\title{
Extending superconducting qubit lifetimes: What's Next?
}

1. Millisecond coherence in a superconducting qubit

Authors: Aaron Somoroff, Quentin Ficheux, Raymond A. Mencia, Haonan Xiong, Roman Kuzmin, and Vladimir E. Manucharyan

arXiv:2103.08578

2. New material platform for superconducting transmon qubits with coherence times exceeding $\mathbf{0 . 3}$ milliseconds

Authors: Alexander P. M. Place, Lila V. H. Rodgers, Pranav Mundada, Basil M.

Smitham, Mattias Fitzpatrick, Zhaoqi Leng, Anjali Premkumar, Jacob Bryon, Andrei

Vrajitoarea, Sara Sussman, Guangming Cheng, Trisha Madhavan, Harshvardhan K.

Babla, Xuan Hoang Le, Youqi Gang, Berthold Jäck, András Gyenis, Nan Yao,

Robert J. Cava, Nathalie P. de Leon, and Andrew A. Houck

Nature Communications 12 (2021)

3. Protecting a bosonic qubit with autonomous quantum error correction Authors: Jeffrey M. Gertler, Brian Baker, Juliang Li, Shruti Shirol, Jens Koch, and Chen Wang

Nature 590, 243 (2021)

Recommended with a Commentary by Steven M. Girvin, Yale University

\section{Introduction}

The coherence times of superconducting qubits have risen approximately five orders of magnitude [1,2] since 1998 when the first such qubit was measured by Nakamura and collaborators [3]. This is fantastic progress but decoherence is still a major issue preventing us from escaping the NISQ (noisy intermediate-scale quantum) era and moving into the era in which we achieve practical quantum advantage on economically and scientifically useful tasks.

Most of the progress to date has been achieved through creation of new qubit designs whose quantum states are intrinsically less sensitive to environmental noise and such work continues [4]. Somoroff et al. use a novel 'fluxonium' low-frequency qubit design that has for the first time achieved coherence times exceeding one millisecond.

Until recently, little serious materials science has been done to find and reduce sources of noise in the devices, though careful systematic experiments and modeling [5-7] have 
demonstrated that it is advantageous to design qubit excitation modes to keep electric fields away from the surfaces of dielectric substrates as much as possible. It seems clear that surfaces of dielectrics are generally much lossier than the bulk. This is presumably from TLS (two-level) defect systems [8] associated with the growth, cutting and polishing the materials (or possibly surface adsorbates which have also been implicated in magnetic flux noise $[9,10])$, but there is little in the way of detailed theoretical understanding of these microscopic defects and their ability to absorb relatively low frequency $(\mathrm{GHz})$ photons. The work of Place et al. nicely illustrates that the situation is now changing as materials scientists begin to collaborate with experts on qubit design to achieve significantly enhanced qubit coherence times.

Nevertheless, no matter how great the gains in physical qubit lifetime are, we are unlikely to ever get around the golden rule: 'There is no such thing as too much coherence.' In order to achieve useful quantum advantage, we will need to run algorithms requiring large circuit depth (many operations). Thus fault-tolerant quantum error correction (roughly speaking, the ability to correct quantum errors even though the correction processes themselves make errors) is the grand challenge for the field. We are just entering the era of quantum error correction (QEC) and beginning to design correction circuits that are tolerant of some types of faults. The work by Gertler et al. nicely illustrates progress on this problem.

\section{Millisecond coherence in a superconducting qubit}

The fluxonium qubit [11] is essentially a small Josephson junction qubit in parallel with a 'superinductor' (comprising a series array of hundreds of Josephson junction) having a reactive impedance comparable to the quantum of impedance $\left(h /\left(4 e^{2}\right)\right)$ that shorts out deleterious low-frequency charge noise but not the high-frequency qubit oscillations. The fluxonium has a so-called $\Lambda$ structure in its spectrum with two low-lying levels and a large gap to the third level. Somoroff et al. report a best-case Ramsey-fringe (without echo) coherence time of $T_{2}=1.48 \pm 0.13 \mathrm{~ms}$ and a similar energy relaxation time $T_{1}$, and a longterm average case $T_{2}=1.16 \pm 0.05 \mathrm{~ms}$, with relatively stable $T_{1}$ values over a period of months. This substantially exceeds previous records for coherence times. However, it is important to note that at the magnetic flux 'sweet spot,' the transition frequency between the two lowest states in the $\Lambda$ structure is only $163 \mathrm{MHz}$, about 30 times lower than typical transmon qubits. Thus $Q$ (the quality factor) has not been noticeably increased, suggesting that materials loss parameters are similar to previous works. Nevertheless, the long coherence time is very helpful for memory purposes and for increasing gate fidelities. Using randomized benchmarking, the authors report single-qubit gate fidelities of 0.9999, a record that has only been exceeded in some ion trap experiments.

\section{New material platform for superconducting transmon qubits}

Place et al. have replaced $\mathrm{Nb}$ in the transmon qubit antenna leads (capacitor) with Ta and improved $T_{1}$ and $T_{2}$ by $\sim 2-3 \mathrm{x}$ to $0.2-0.3 \mathrm{~ms}$. Application of a variety of microscopic materials characterization and imaging tools (atomic-resolution TEM, x-ray diffraction, XPS, ARPES) indicates that Ta offers a simpler oxide than $\mathrm{Nb}$ and that single-crystal columns of Ta grow epitaxially on the sapphire substrate. Qubit coherence measurements suggest that the dissipation is low despite the columnar growth structure. Ta also offers the advantage that it is more robust to aggressive cleaning and surface preparation than traditional allaluminum devices. (In the Ta devices, the Ta antenna pads are deposited and cleaned, and 
then an Al Josephson junction is added to connect the two pads.) Much work remains to be done to connect processing conditions to both microscopic structure and macroscopic device performance, but this paper illuminates the route ahead.

\section{Protecting a bosonic qubit with autonomous quantum error correction}

At first sight quantum error correction seems impossible because of the no-go theorem for correcting errors in analog computers. However, while quantum states and their errors are indeed analog, measured errors are (because of state collapse) discrete. This miracle makes quantum error correction possible, in principle. In practice, quantum error correction is fantastically difficult. In traditional qubit-based codes one encodes the logical information in entangled states of $N$ physical qubits. This makes the physical error rate $N$ times worse (assuming uncorrelated errors) but allows for the possibility of making special error syndrome measurements which partially collapse the state, revealing which error has occurred on which of the $N$ qubits, without revealing (and thereby destroying) the logical state in which the error occurred. The 'Maxwell demon' that measures and corrects the errors must be very fast, very accurate, and be non-demolition in order to overcome the factor of $N$ in the error rate to reach the break-even point where quantum error correction begins to enhance the lifetime of the quantum information. This has not yet been achieved with superconducting qubits but is a subject of intense work (see for example, Ref. [12]).

Bosonic encoding [13-16] offers a number of crucial advantages over qubit encodings and there are now several experiments [17-21] that have approached or slightly exceeded the break-even point. In bosonic encodings, the logical quantum information is encoded in superpositions of different Fock states (of microwave photons stored in superconducting cavities, or in the mechanical oscillations of trapped ions or trapped phonons in solids). The advantage of bosonic encoding is that it is relatively easy to store bosons for extended periods and the error channel is typically simple. For superconducting resonators it is often simple amplitude damping with negligible intrinsic dephasing (frequency fluctuations of the resonator). Furthermore, the amplitude damping occurs in a single mode in contrast to the amplitude damping in $N$ physical qubits which occurs in $N$ different physical locations that must be determined through measurement of complex (i.e. high-weight) stabilizer operators. In addition to amplitude damping, qubits also suffer from dephasing and other errors.

One might wonder how unitary error correction operations can reduce the entropy in a logical qubit. This happens because the particular unitary operation that is performed to correct an error is conditioned on the outcome of the measurement of the error syndromes. Thus the overall correction process is non-unitary and entropy changing. An alternative approach to measurement-based error correction is autonomous error correction. In this case one uses quantum noise engineering to create novel cold baths which dissipate the entropy by 'cooling' the logical qubit into the error-free subspace. One advantage of autonomous schemes is that they involve simply turning on constant microwave drives to engineer the desired dissipation. Unlike measurement-based systems, it is not necessary to relay measurement information up from the cryostat to room temperature electronics to make a decision on what control pulse should next be sent down into the cryostat.

Gertler et al. have made a remarkable advance in autonomous error correction using a so-called 'Truncated 4-component Cat' (T4C) bosonic code. Here the code words are two orthogonal linear combinations of photon Fock states with $n=1,3,5,7$. Since the code 
words all have odd parity, photon loss could be detected by measuring the photon number parity-something that was done in the first bosonic error correction experiment using the cat code [17]. However, rather than measure the photon loss and then attempt to repair it, the authors developed a novel and rather sophisticated bath engineering scheme that autonomously adds back a photon every time one is lost. This is a non-trivial task, not in the least because all the 'which path information' must be erased in order to prevent the environment from learning anything about the particular logical state (linear combination of code words) stored in the cavity. The 'which path' information leaks into the environment through the fact the matrix elements for destroying and creating photons in Fock state $n$, depend on $n$ through the $\sqrt{n}$ and $\sqrt{n+1}$ matrix elements. The protocol used by the authors cleverly circumvents these issues through careful engineering of the drives on the system that induce the desired dissipation. Without this erasure, the quantum information stored in the logical qubit would collapse. We are just at the beginning of the era of quantum error correction and much work remains to be done, but this work is an interesting step forward.

As a final side note, it is interesting to see that qubit coherence times have gotten long enough that in the near future, cosmic rays and radioactive decay events may become a limiting factor that will require amelioration [22]. A number of recent experiments in MKIDs (bolometers based on kinetic inductance changes) $[23,24]$ and in superconducting qubits and qubit arrays [25-27] have seen convincing evidence for cosmic ray energy deposition causing burst events in which one or multiple qubits temporarily fail due to an excess of broken Cooper pairs created by the energy deposition. Correlated errors, which are especially are dangerous for quantum error correction, come about across large distances because it is hard for the superconducting quasiparticles to thermalize and fall back into the superconducting condensate. When they do recombine, phonons of energy at least twice the superconducting gap are released and can travel through the substrate to distant qubits where they are absorbed and turn back into broken Cooper pairs causing further damage.

Remarkably, it is now possible to experimentally to detect single quasiparticles tunneling through Josephson junctions and see their effect on qubit relaxation (in both energy and phase) [28-30]. A useful review on the topic is Ref. [31]. It is now understood that excess non-equilibrium quasiparticles are abundant and there are now good indications that they are associated with stray millimeter- or far-infrared photons leaking past microwave filters/attenuators whose efficiency is poor at high frequencies [32]. Once we find ways to clean these up (through 'microwave hygiene') and further reduce substrate dielectric loss another order of magnitude through modern materials science, cosmic rays may be the next culprit. It's always something...

This work was supported by the DOE Office of Science National Quantum Information Science Research Centers, Co-design Center for Quantum Advantage (C2QA) under contract number DE-SC0012704.

\section{References}

[1] M. H. Devoret and R. J. Schoelkopf. Superconducting circuits for quantum information: An outlook. Science, 339(6124):1169-1174, 2013. 
[2] William D. Oliver and Paul B. Welander. Materials in superconducting quantum bits. MRS Bulletin, 38:816, 2013.

[3] Y. Nakamura, Yu A. Pashkin, and J. S. Tsai. Coherent control of macroscopic quantum states in a single-cooper-pair box. Nature, 398(6730):786-788, 1999.

[4] András Gyenis, Agustin Di Paolo, Jens Koch, Alexandre Blais, Andrew A. Houck, and David I. Schuster. Moving beyond the transmon: Noise-protected superconducting quantum circuits. PRX Quantum, 2:030101, Sep 2021.

[5] Hanhee Paik, D. I. Schuster, Lev S. Bishop, G. Kirchmair, G. Catelani, A. P. Sears, B. R. Johnson, M. J. Reagor, L. Frunzio, L. I. Glazman, S. M. Girvin, M. H. Devoret, and R. J. Schoelkopf. Observation of high coherence in josephson junction qubits measured in a three-dimensional circuit qed architecture. Phys. Rev. Lett., 107:240501, Dec 2011.

[6] R. Barends, J. Kelly, A. Megrant, D. Sank, E. Jeffrey, Y. Chen, Y. Yin, B. Chiaro, J. Mutus, C. Neill, P. O’Malley, P. Roushan, J. Wenner, T. C. White, A. N. Cleland, and John M. Martinis. Coherent josephson qubit suitable for scalable quantum integrated circuits. Phys. Rev. Lett., 111:080502, Aug 2013.

[7] C. Wang, C. Axline, Y. Y. Gao, T. Brecht, Y. Chu, L. Frunzio, M. H. Devoret, and R. J. Schoelkopf. Surface participation and dielectric loss in superconducting qubits. Applied Physics Letters, 107(16):162601, 2015.

[8] Daniel M. Tennant, Luis A. Martinez, Chris D. Wilen, Robert McDermott, Jonathan L DuBois, and Yaniv J. Rosen. Low frequency correlated charge noise measurements across multiple energy transitions in a tantalum transmon. arXiv:2106.08406, 2021.

[9] P. Kumar, S. Sendelbach, M. A. Beck, J. W. Freeland, Zhe Wang, Hui Wang, Clare C. $\mathrm{Yu}, \mathrm{R}$. Q. Wu, D. P. Pappas, and R. McDermott. Origin and reduction of $1 / f$ magnetic flux noise in superconducting devices. Phys. Rev. Applied, 6:041001, Oct 2016.

[10] Jochen Braumüller, Leon Ding, Antti P. Vepsäläinen, Youngkyu Sung, Morten Kjaergaard, Tim Menke, Roni Winik, David Kim, Bethany M. Niedzielski, Alexander Melville, Jonilyn L. Yoder, Cyrus F. Hirjibehedin, Terry P. Orlando, Simon Gustavsson, and William D. Oliver. Characterizing and optimizing qubit coherence based on squid geometry. Phys. Rev. Applied, 13:054079, May 2020.

[11] Vladimir E. Manucharyan, Jens Koch, Leonid I. Glazman, and Michel H. Devoret. Fluxonium: Single cooper-pair circuit free of charge offsets. Science, 326(5949):113$116,2009$.

[12] Zijun Chen et al. Exponential suppression of bit or phase flip errors with repetitive error correction. arXiv:2102.06132, 2021.

[13] Mazyar Mirrahimi, Zaki Leghtas, Victor V. Albert, Steven Touzard, Robert J. Schoelkopf, Liang Jiang, and Michel H. Devoret. Dynamically protected cat-qubits: a new paradigm for universal quantum computation. New Journal of Physics, 16(4):045014, 2014. 
[14] Atharv Joshi, Kyungjoo Noh, and Yvonne Y Gao. Quantum information processing with bosonic qubits in circuit QED. Quantum Science and Technology, 6(3):033001, apr 2021.

[15] Alexandre Blais, Arne L. Grimsmo, S. M. Girvin, and Andreas Wallraff. Circuit quantum electrodynamics. Rev. Mod. Phys., 93:025005, May 2021.

[16] Alexandre Blais, Steven M. Girvin, and William D. Oliver. Quantum information processing and quantum optics with circuit quantum electrodynamics. Nature Physics, 16(3):247-256, 2020.

[17] Nissim Ofek, Andrei Petrenko, Reinier Heeres, Philip Reinhold, Zaki Leghtas, Brian Vlastakis, Yehan Liu, Luigi Frunzio, S. M. Girvin, L. Jiang, Mazyar Mirrahimi, M. H. Devoret, and R. J. Schoelkopf. Extending the lifetime of a quantum bit with error correction in superconducting circuits. Nature, 536(7617):441-445, 2016.

[18] L. Hu, Y. Ma, W. Cai, X. Mu, Y. Xu, W. Wang, Y. Wu, H. Wang, Y. P. Song, C. L. Zou, S. M. Girvin, L. M. Duan, and L. Sun. Quantum error correction and universal gate set operation on a binomial bosonic logical qubit. Nature Physics, 15(5):503-508, 2019.

[19] Philip Reinhold, Serge Rosenblum, Wen-Long Ma, Luigi Frunzio, Liang Jiang, and Robert J. Schoelkopf. Error-corrected gates on an encoded qubit. Nature Physics, 16(8):822-826, 2020.

[20] P. Campagne-Ibarcq, A. Eickbusch, S. Touzard, E. Zalys-Geller, N. E. Frattini, V. V. Sivak, P. Reinhold, S. Puri, S. Shankar, R. J. Schoelkopf, L. Frunzio, M. Mirrahimi, and M. H. Devoret. Quantum error correction of a qubit encoded in grid states of an oscillator. Nature, 584(7821):368-372, 2020.

[21] Brennan de Neeve, Thanh Long Nguyen, Tanja Behrle, and Jonathan Home. Error correction of a logical grid state qubit by dissipative pumping. arXiv:2010.09681, 2020.

[22] John M. Martinis. Saving superconducting quantum processors from decay and correlated errors generated by gamma and cosmic rays. npj Quantum Information, 7(1):90, 2021.

[23] K. Karatsu, A. Endo, J. Bueno, P. J. de Visser, R. Barends, D. J. Thoen, V. Murugesan, N. Tomita, and J. J. A. Baselmans. Mitigation of cosmic ray effect on microwave kinetic inductance detector arrays. Applied Physics Letters, 114(3):032601, 2019.

[24] L. Cardani, N. Casali, G. Catelani, T. Charpentier, M. Clemenza, I. Colantoni, A. Cruciani, L. Gironi, L. Gruenhaupt, D. Gusenkova, F. Henriques, M. Lagoin, M. Martinez, S. Pirro, I. M. Pop, C. Rusconi, A. Ustinov, F. Valenti, M. Vignati, and W. Wernsdorfer. Demetra: Suppression of the relaxation induced by radioactivity in superconducting qubits. Journal of Low Temperature Physics, 199(1):475-481, 2020. 
[25] Antti P. Vepsäläinen, Amir H. Karamlou, John L. Orrell, Akshunna S. Dogra, Ben Loer, Francisca Vasconcelos, David K. Kim, Alexander J. Melville, Bethany M. Niedzielski, Jonilyn L. Yoder, Simon Gustavsson, Joseph A. Formaggio, Brent A. VanDevender, and William D. Oliver. Impact of ionizing radiation on superconducting qubit coherence. Nature, 584(7822):551-556, 2020.

[26] C. D. Wilen, S. Abdullah, N. A. Kurinsky, C. Stanford, L. Cardani, G. D’Imperio, C. Tomei, L. Faoro, L. B. Ioffe, C. H. Liu, A. Opremcak, B. G. Christensen, J. L. DuBois, and R. McDermott. Correlated charge noise and relaxation errors in superconducting qubits. Nature, 594(7863):369-373, 2021.

[27] Matt McEwen, Lara Faoro, Kunal Arya, Andrew Dunsworth, Trent Huang, Seon Kim, Brian Burkett, Austin Fowler, Frank Arute, Joseph C. Bardin, Andreas Bengtsson, Alexander Bilmes, Bob B. Buckley, Nicholas Bushnell, Zijun Chen, Roberto Collins, Sean Demura, Alan R. Derk, Catherine Erickson, Marissa Giustina, Sean D. Harrington, Sabrina Hong, Evan Jeffrey, Julian Kelly, Paul V. Klimov, Fedor Kostritsa, Pavel Laptev, Aditya Locharla, Xiao Mi, Kevin C. Miao, Shirin Montazeri, Josh Mutus, Ofer Naaman, Matthew Neeley, Charles Neill, Alex Opremcak, Chris Quintana, Nicholas Redd, Pedram Roushan, Daniel Sank, Kevin J. Satzinger, Vladimir Shvarts, Theodore White, Z. Jamie Yao, Ping Yeh, Juhwan Yoo, Yu Chen, Vadim Smelyanskiy, John M. Martinis, Hartmut Neven, Anthony Megrant, Lev Ioffe, and Rami Barends. Resolving catastrophic error bursts from cosmic rays in large arrays of superconducting qubits. arXiv:2104.05219, 2021.

[28] G. Catelani, R. J. Schoelkopf, M. H. Devoret, and L. I. Glazman. Relaxation and frequency shifts induced by quasiparticles in superconducting qubits. Phys. Rev. B, 84:064517, Aug 2011.

[29] Ioan M. Pop, Kurtis Geerlings, Gianluigi Catelani, Robert J. Schoelkopf, Leonid I. Glazman, and Michel H. Devoret. Coherent suppression of electromagnetic dissipation due to superconducting quasiparticles. Nature, 508(7496):369-372, 2014.

[30] C. Wang, Y. Y. Gao, I. M. Pop, U. Vool, C. Axline, T. Brecht, R. W. Heeres, L. Frunzio, M. H. Devoret, G. Catelani, L. I. Glazman, and R. J. Schoelkopf. Measurement and control of quasiparticle dynamics in a superconducting qubit. Nature Communications, 5(1):5836, 2014.

[31] Leonid I. Glazman and Gianluigi Catelani. Bogoliubov Quasiparticles in Superconducting Qubits. SciPost Phys. Lect. Notes, page 31, 2021.

[32] M. Houzet, K. Serniak, G. Catelani, M. H. Devoret, and L. I. Glazman. Photon-assisted charge-parity jumps in a superconducting qubit. Phys. Rev. Lett., 123:107704, Sep 2019. 